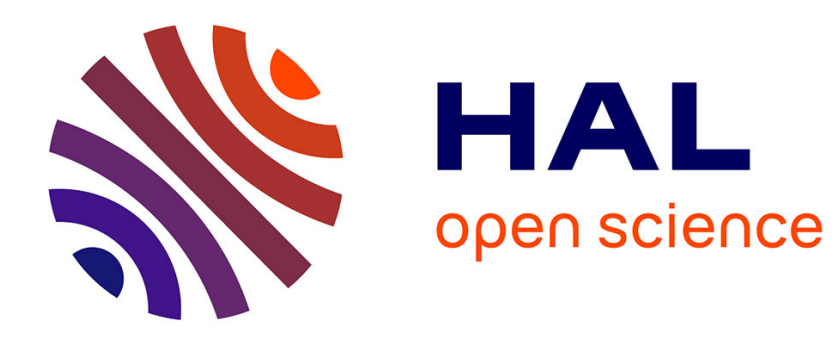

\title{
Rôle des sémiochimiques dans les relations pucerons-plantes. II. - Les substances allélochimiques
}

\author{
Etienne Herrbach
}

\section{To cite this version:}

Etienne Herrbach. Rôle des sémiochimiques dans les relations pucerons-plantes. II. - Les substances allélochimiques. Agronomie, 1985, 5 (4), pp.375-384. hal-00884772

\section{HAL Id: hal-00884772}

\section{https://hal.science/hal-00884772}

Submitted on 1 Jan 1985

HAL is a multi-disciplinary open access archive for the deposit and dissemination of scientific research documents, whether they are published or not. The documents may come from teaching and research institutions in France or abroad, or from public or private research centers.
L'archive ouverte pluridisciplinaire HAL, est destinée au dépôt et à la diffusion de documents scientifiques de niveau recherche, publiés ou non, émanant des établissements d'enseignement et de recherche français ou étrangers, des laboratoires publics ou privés. 


\section{Rôle des sémiochimiques dans les relations puce- rons-plantes. II. - Les substances allélochimi- ques}

Etienne HERRBACH

I.N.R.A., Station de Zoologie, Route de Herrlisheim, F-68021 Colmar

RÉSUMÉ

Les relations plantes-insectes dépendent de divers facteurs dont des substances chimiques, les allélochimiques. Le présent travail est une mise au point bibliographique sur les effets des allélochimiques d'origine végétale sur le comportement aphidien. L'accent est mis sur le rôle de ces substances dans la spécificité des relations pucerons-plantes et dans le déroulement des processus du choix de l'hôte et de l'alimentation. L'utilisation possible des allélochimiques comme moyens de lutte contre les pucerons et la propagation de viroses végétales est discutée.

Mots clés additionnels : Choix plante-hôte, communication chimique, comportement alimentaire, Homoptera Aphididae, transmission de virus.

The relationships between phytophagous insects and their host are controlled by numerous factors. Among these, allelochemicals can influence insect feeding behaviour. In the present work, the effects of plant allelochemicals on aphid behaviour are reviewed. Emphasis is put on the rôle of these substances in the specificity of aphid-plant relationships and in the host selection and feeding processes. It is suggested that allelochemicals could be used as an alternative means for controlling aphid colonisation and the spread of plant viruses.

Additional key words : Chemical communication, feeding behaviour, Homoptera Aphididae, host plant selection, virus transmission.

\section{INTRODUCTION}

Le comportement des pucerons est influencé par des substances chimiques, les sémiochimiques. Dans une $1^{\text {re }}$ partie (HERRBACH, 1985), nous avons évoqué l'influence des phéromones d'alarme des pucerons. Dans cette $2^{\mathrm{c}}$ partie, nous présentons une synthèse des connaissances actuelles sur le rôle des allélochimiques dans le comportement alimentaire des pucerons. La démarche adoptée consiste à inventorier les allélochimiques par classes chimiques, en essayant autant que possible de dégager l'importance de ces substances dans les processus de choix de la plante-hôte et d'alimentation des pucerons.
Chez les pucerons, le comportement de sélection de l'hôte et d'alimentation se déroule selon la séquence : atterrissage des ailés - contact tactile - piqûre (s) d'épreuve - pénétration des stylets - ingestion de sève. Les ailés visitent de nombreuses plantes et la sélection s'opère sur la base de divers facteurs physiques et chimiques. Les facteurs chimiques comprennent des substances stimulantes ou inhibitrices. Des nutriments essentiels (aminoacides, sucres) déclenchent souvent un effet comportemental, indépendamment de leur valeur nutritive propre. Nous pensons, avec MASSON (1982), devoir classer de telles substances parmi les allélochimiques dès lors qu'un effet comportemental est engendré. 


\section{INVENTAIRE ET RÔLE DES ALLÉLOCHIMIQUES}

\section{A. Aminoacides}

En solution nutritive ou dans le végétal, les aminoacides protéiques présentent des effets multiples sur les pucerons. Leur influence sur la croissance et la fécondité a été soulignée (VAN EMDEN \& BASHFORD, 1971 ; VAN EMDEN, 1973). Plusieurs aminoacides possèdent une valeur phagostimulante et, de ce fait, agissent sur le comportement de sélection de la plante-hôte et d'alimentation.

Pour Myzus persicae (Sulzer), placé en situation de choix (MITTLER \& DADD, 1964) ou en situation de non-choix (MITTLER \& DADD, 1965), une solution de saccharose contenant un mélange de $6 \mathrm{~L}$-aminoacides (asparagine, leucine, lysine, méthionine, thréonine, valine) est nettement plus stimulante qu'une simple solution de saccharose; par contre, une solution de ces aminoacides n'est pas plus stimulante que l'eau. La valeur phagostimulante d'une solution nutritive est fonction de sa concentration en aminoacides, elle passe par un optimum ( 2 à 3 p. 100 du milieu) et décroît au-delà (MitTler, 1967a ; SRIVASTAVA \& AUCLAIR, 1974).

La valeur phagostimulante d'un milieu synthétique est liée non seulement à la teneur globale en aminoacides et à leur effet de synergie avec le saccharose, mais encore à la présence de certains aminoacides particuliers.

La préférence de $M$. persicae pour une solution de saccharose est fortement augmentée par chacun de ces 6 aminoacides : méthionine, phénylalanine, leucine, isoleucine, tryptophane, asparagine, tandis que l'histidine, l'acide glutamique et l'arginine sont légèrement répulsifs (MITTLER, 1967b). LECKSTEIN \& LLEWELLYN (1974) établissent qu'Aphis fabae Scopoli préfère un milieu contenant de la méthionine $(l$, fig. 1) ou de la proline, à un milieu qui en est dépourvu. Acyrthosiphon pisum (Harris) est sensible aux aminoacides au moment de la $1^{\text {re }}$ piqûre d'épreuve. La durée de celle-ci est augmentée par 4 aminoacides (acide aspartique, isoleucine, valine, leucine) et réduite par 5 autres (phénylalanine, arginine, tryptophane, cystéine, thréonine) (KLINGAUF \& NÖCKER-WENZEL, 1972).

Par mesure du taux d'ingestion, on a mis en évidence le pouvoir phagostimulant de plusieurs aminoacides : la méthionine (MITTLER, 1967c, 1970), la cystéine et l'histidine (MITTLER, 1970) pour M. persicae, la méthionine pour $A$. pisum (Srivastava \& AuClaIR, 1974).

$\mathrm{La}$ relation entre le pouvoir phagostimulant des aminoacides et leur caractère essentiel à l'entretien et au développement des pucerons (AUCLAIR, 1969) reste mal comprise. Le caractère essentiel d'un aminoacide dépend de la présence dans le milieu d'autres composés (MASSONIÉ, 1973). Selon LeCKSTEIN \& LLEWELLYN (1974), l'histidine et la méthionine, essentielles pour $A$. fabae, ne sont pas phagostimulantes, alors que l'alanine et la proline, non essentielles, sont phagostimulantes. La méthionine, l'histidine et l'isoleu- cine sont essentielles au développement de $M$. persicae sur milieu synthétique (DADD \& KRIEGER, 1968), mais seule la méthionine serait phagostimulante pour cette espèce (HARREWIJN \& NOORDINK, 1971).

La composition en aminoacides des tissus végétaux superficiels entre en jeu dans le déclenchement du comportement alimentaire d'A. fabae par stimulation de la $1^{\text {re }}$ piqûre d'épreuve. Toutefois cette composition n'a pas valeur d'information sur le statut d'hôte de la plante ; en effet des mélanges d'aminoacides protéiques dans les proportions trouvées naturellement dans les tissus superficiels de Galinsoga parviflora Cavanilles, composée non-hôte pour $A$. fabae, provoquent la même stimulation que ceux des hôtes Vicia faba L. et Beta vulgaris L. (JÖRDENS-RÖTTGER, 1979a). En revanche, il est établi qu'un aminoacide non protéique, la L-DOPA (L-dihydroxy-3,4 phénylalanine) $(2$, fig. 1), extraite de $V$. $f a b a$, est déterminant dans la reconnaissance de cette plante par $A$. fabae (JÖRDENS \& KLINGAUF, 1977 ; JöRDENS-RöTTGER, 1979a).

Les expérimentations en solutions nutritives ont mis en évidence le pouvoir phagostimulant des aminoacides sur les pucerons. Le pouvoir phagostimulant d'un aminoacide donné peut varier selon son mode de présentation au puceron et le type de réponse enregistrée; il en est ainsi de l'histidine pour $M$. persicae (MitTler, 1967b, 1970 ; HARREWIJN \& NOORDINK, 1971). La compréhension du rôle des aminoacides dans les relations entre pucerons et plante-hôte demeure imparfaite. Les aminoacides interviennent dans le déclenchement de la prise alimentaire sur végétal chez A. fabae. Par leur pouvoir phagostimulant et leur qualité de nutriments, ils influencent la poursuite du comportement alimentaire, en jouant à la fois sur la valeur phagostimulante et la qualité nutritionnelle de la plante (VAN EMDEN, 1972).

\section{B. Sucres}

Le saccharose est un des phagostimulants les plus universels (BERNAYS \& SIMPSON, 1982), il stimule la prise alimentaire de plusieurs pucerons : $M$. persicae (MITTLER \& DADD, 1964, 1965), Brevicoryne brassicae (L.) (MOON, 1967), A. pisum (AUCLAIR, 1969 ; SRIVASTAVA \& AUClair, 1971), Amphorophora agathonica Hottes (ARN \& CLEERE, 1971). Cependant les fortes concentrations (supérieures à $10 \mathrm{p} .100$ ) peuvent réduire le taux d'alimentation de $M$. persicae (MITTLER, 1967a).

D'autres sucres (mélézitose, raffinose, glucose, galactose, maltose, tréhalose, sorbose) stimulent l'ingestion chez $M$. persicae, généralement en synergie avec le saccharose, tandis que le mannose, le ribose, le rhamnose, et l'arabinose sont plutôt phagodissuadants (MITTLER et al., 1970).

Le sorbitol (3, fig. 1) est un sucre-alcool présent chez certaines rosacées. Sans être stimulant, il est toléré par Aphis pomi de Geer, strictement inféodé aux espèces contenant du sorbitol. Il agit comme phagodissuadant sur $A$. agathonica, qui refuse les espèces à sorbitol, et sur $M$. persicae, qui cependant accepte le sorbitol en milieu artificiel après une période d'accomodation (MITTLER et al., 1970 ; MONTGOMERY, 1975). 


$$
\begin{array}{r}
\mathrm{CH}_{3}-\mathrm{S}-\left(\mathrm{CH}_{2}\right)_{2}-\mathrm{CH}-\mathrm{NH}_{2} \\
\mathrm{C} \\
\mathrm{COOH}
\end{array}
$$

Méthionine

1<smiles>N[C](Cc1ccc(O)c(O)c1)C(=O)O</smiles>

$\underset{\substack{\text { L DOPA } \\ \underline{2}}}{ }$

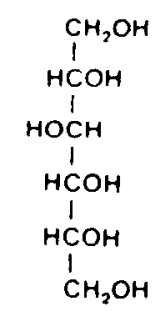

Sorbitol

$\underline{3}$<smiles>COOC(C#N)c1ccc(O)cc1</smiles>

Amygdaline

$\underline{6}$

$\mathrm{CH}_{2}=\mathrm{CH}-\mathrm{CH}_{2}-\underset{\mathrm{N}}{\mathrm{C}}-\mathrm{S}-\mathrm{OS}$

Sinigrine

$\underline{9}$<smiles>O=c1cc(-c2ccc(O)c(O)c2)oc2cc(O)cc(O)c12</smiles>

Lutéoline<smiles>COc1ccc2c(c1)OC(O)C(=O)N2O</smiles>

DIMBOA

?

$\mathrm{CH}_{3}-\left(\mathrm{CH}_{2}\right)_{20}-\mathrm{COOH}$

Acide dodécanoïque

10

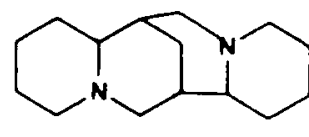

Spartéine

$\underline{13}$

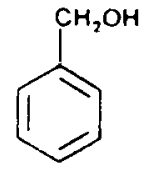

Alcool benzylique 14

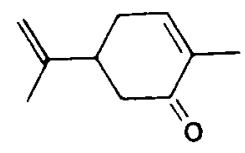

Carvone

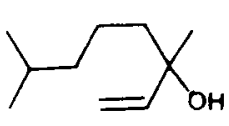

Linalool

16<smiles>CC1(C)CCCC2(C)C(C=O)C(C=O)=CCC12</smiles>

Polygodial

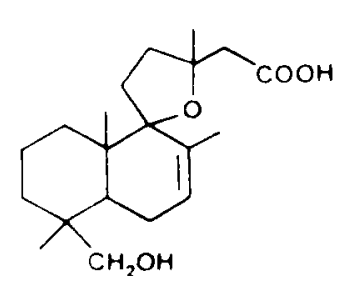

Acide hydroxygrindélique

$\underline{18}$

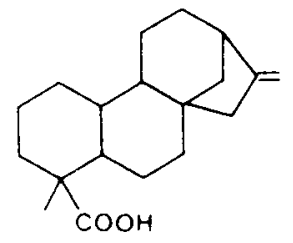

Acide kaurénoïque

$\underline{19}$

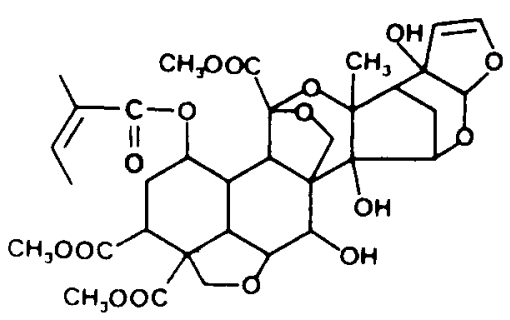

Azadirachtine

$\underline{20}$<smiles>O=c1ccc2ccccc2o1</smiles>

Coumarine $\underline{21}$

Figure 1

Formules développées d'allélochimiques cités dans le texte.

Molecular structures of allelochemicals quoted in text. 


\section{Glycosides}

Plusieurs glycosides agissent sur la prise de nourriture des pucerons. Souvent l'aglycone seul présente une activité biologique sur le phytophage. C'est le cas de la lutéoline (4, fig. 1), flavone phagostimulante pour $A$. fabae (JÖRDENS-RÖTTGER, 1979b) et antiappétante pour Schizaphis graminum (Rondani) (DREYER \& JONES, 1981), alors que le lutéoline 7-0 glucoside est inactif sur $S$. graminum (DREYER et al., 1981 ; DREYER \& JONES, 1981). On connaît l'activité antiappétante sur $S$. graminum de 2 glycosides cyanogènes, la dhurrine $\left(5\right.$, fig. 1) $\left(\operatorname{ED}_{50}\left(^{*}\right)\right.$ de $0,16 \mathrm{p}$. 100) et l'amygdaline $(6$, fig. 1$)\left(E_{50}\right.$ de 0,5 p. 100) (DREYER et al., 1981), cette dernière également active à $0,01 \mathrm{M}$ sur $M$. persicae (SCHOONHOVEN \& DERKSENKOPPERS, 1976).

Citons encore le DIMBOA (dihydroxy-2,4 méthoxy7 benzoxazine-1,4 one-3) (7, fig. 1), aglycone d'un glucoside présent dans le maïs et d'autres céréales; son action létale sur Rhopalosiphum maidis (Fitch), Metopolophium dirhodum (Walker) et $S$. graminum (LONG et al., 1977 ; ARGANDOŇA et al., 1980, 1981 ; CORCUERA et al., 1982) s'accompagne d'un effet phagodissuadant (ARGANDOÑA et al., 1983).

Nous nous arrêterons à 2 glucosides (glycosides du glucose), la phlorizine et la sinigrine, très étudiés et intéressants au point de vue de la relation allélochimique-spécificité.

\section{La phlorizine}

La phlorizine ( 8 , fig. 1), glucoside phénolique spécifique du genre Malus (pommier) et de quelques autres espèces de Pomoidae, est un exemple d'allélochimique agissant à plusieurs niveaux du comportement de sélection de certaines espèces de pucerons, en relation avec la spécificité de celles-ci.

Le comportement d'épreuve sur milieu artificiel d'Aphis pomi et de Rhopalosiphum insertum (Walker), 2 espèces associées au genre Malus, est stimulé par la phlorizine (à 0,005 p. 100). Pour ces espèces, la phlorizine agit comme arrestant (KLINGAUF, 1971).

La phlorizine inhibe le comportement d'épreuve d'espèces non inféodées aux Pomoidae: Acyrthosiphon pisum (KLINGAUF, 1971), Amphorophora agathonica vivant sur Rubus spp. et $M$. persicae (MONTGOMERY \& ARN, 1974 ; MONTGOMERY, 1975 ; SCHOONHOVEN \& DERKSEN-KOPPERS, 1976). Les phases de pénétration et d'ingestion de ces espèces sont réduites par la phlorizine et par son aglycone, la phlorétine (dihydrochalcone) (MONTGOMERY \& ARN, 1974 ; MONTGOMERY, 1975 ; DREYER \& JONES, 1981). L'inhibition de la pénétration et de l'ingestion sur milieu artificiel se produit également chez $A$. pomi (MONTGOMERY \& ARN, 1974).

La réponse comportementale d' $A$. pomi à la phlorizine montre qu'un allélochimique peut avoir un effet

(*) $\mathrm{ED}_{50}=$ concentration de substance dans le milieu provoquant une réduction de 50 p. 100 du nombre de pucerons s'alimentant, par rapport au témoin. positif sur la phase d'épreuve et un effet négatif sur la phase d'ingestion, le premier effet intervenant directement dans la reconnaissance de l'hôte. Le second effet ne compromet pas l'alimentation normale d' $A$. pomi, car le phloème où le puceron se nourrit semble dépourvu de phlorizine (MONTGOMERY \& ARN, 1974).

\section{La sinigrine}

Les glucosinolates sont des glucosides soufrés, omniprésents dans la famille des Crucifères et quelques familles voisines (UNDERHILL, 1980). Le rôle joué par un glucosinolate, la sinigrine $(9$, fig. 1), et son aglycone, l'allylisothiocyanate, sur le comportement de sélection de l'hôte de nombreux insectes est désormais bien établi (WHITTAKER \& FEENY, 1971 ; LÉRIN, 1980). Pour exposer la réponse des pucerons à la sinigrine, nous distinguerons 3 types de spécificité.

\section{a) Cas des espèces oligophages inféodées aux Crucifères}

L'expérience de WENSLER (1962) constitue la première mise en évidence du rôle de la sinigrine comme stimulus spécifique de la sélection de l'hôte par $B$. brassicae; une plante non-hôte, Vicia faba, ayant absorbé de la sinigrine est acceptée par ce puceron.

La sinigrine affecte plusieurs phases du comportement de sélection de l'hôte par les pucerons. Elle agit, par l'aglycone volatil, comme arrestant pour Lipaphis erysimi (Kaltenbach) (NAULT \& STYER, 1972) et pour B. brassicae (PETTERSSON, 1973) ; en d'autres termes, elle inhibe l'activité de vol des ailés et conduit à la piqûre d'épreuve et à la reconnaissance de la plante-hôte sur la base de la présence ou de l'absence de sinigrine. La présence de sinigrine n'est pas détectée par l'ailé en vol (PETTERSSON, 1979).

La prise alimentaire est influencée par la sinigrine. En solution aqueuse, la sinigrine seule n'est pas stimulante pour $B$. brassicae, mais le devient en présence de saccharose par synergie (MOON, 1967 ; WEARING, 1968 ; KLINGAUF et al., 1972 ; VAN EMDEN, 1972). La sinigrine favorise encore la pénétration des stylets dans une membrane de parafilm (NAULT \& STYER, 1972). L'observation des différentes phases de l'alimentation par la méthode actographique de MAC LEAN \& KINSEY (1964) montre la similitude du comportement de $L$. erysimi sur chou, d'une part, et sur $V$. faba ayant absorbé de la sinigrine, d'autre part (NAULT \& STYER, 1972).

Enfin, la sinigrine agit favorablement sur la longévité et la reproduction de $B$. brassicae, bien après que la sélection de l'hôte se soit opérée (WEARING, 1968 ; VAN EMDEN, 1972, 1973, 1978). Il faut ajouter que $B$. brassicae possède un système enzymatique à base de thioglucosidase, capable d'hydrolyser la sinigrine (MAC GibBon \& Allison, 1968, cités par LÉRIN, 1980).

\section{b) Cas des espèces non inféodées aux Crucifères}

Ajoutée à une solution de saccharose, la sinigrine réduit la prise alimentaire d' $A$. fabae (espèce polyphage rejetant toute crucifère, hormis Capsella bursapastoris Moench), Acyrthosiphon pisum (puceron de légumineuses) et Rhopalosiphom padi (L.) (puceron vivant sur Prunus spp. et sur graminées), mais stimule 
l'ingestion d'un puceron de légumineuses, Megoura viciae Buckton (KLINGAUF et al., 1972). Il est probable que le refus des Crucifères par $M$. viciae repose sur d'autres facteurs que la présence de sinigrine.

Absorbée par Vicia faba, la sinigrine dissuade la fixation d'A. fabae, d'A. pisum, ainsi que celle d'Aulacorthum solani (Kaltenbach), espèce polyphage et occasionnelle sur Crucifères (NAULT \& STYER, 1972).

\section{c) Cas du polyphage Myzus persicae}

$M$. persicae est fréquemment observé sur Crucifères. Sa réponse à la sinigrine varie selon les conditions d'expérimentation :

- la sinigrine stimule la prise alimentaire de $M$. persicae, en solution de saccharose (KLINGAUF et al., 1972 ; KUNKEL, 1977) ou absorbée par $V$. faba (NAULT \& STYER, 1972) ;

- sur aliment synthétique, elle n'est pas préférée par $M$. persicae en situation de choix et est bien tolérée en situation de non-choix (SCHOONHOVEN \& DERKSEN-KOPPERS, 1976 ; QIN \& KE, 1984) ;

- elle présente, selon WEARING (1968), un certain pouvoir phagodissuadant en solution de saccharose ;

- elle provoque à haute concentration un effet dépressif sur la reproduction de $M$. persicae (VAN EMDEN, 1972).

En résumé, il apparaît que l'effet comportemental de la sinigrine sur les pucerons est dépendant de leur association aux Crucifères. Pour les espèces oligophages des Crucifères, la sinigrine agit comme stimulus de reconnaissance de l'hôte et de la prise alimentaire, en synergie avec le saccharose. La sinigrine est antiappétante pour les espèces oligo- et polyphages non inféodées aux Crucifères. La réponse du polyphage $M$. persicae est moins stricte. De manière générale, cette espèce est peu sensible aux allélochimiques non nutritifs (SCHOONHOVEN \& DERKSEN-KOPPERS, 1976 ; VAN EMDEN, 1978 ; QIN \& KE, 1984).

\section{Acides carboxyliques}

L'influence sur le comportement de $M$. persicae de certains acides gras et dérivés est étudiée depuis la mise en évidence de l'effet inhibiteur d'extraits de $M$. persicae (GRIFFITHS et al., 1978), et spécialement des fractions lipidiques (GREENWAY et al., 1978), sur la fixation et le dépôt de larves de ce puceron sur milieu artificiel. On ignore si ces molécules remplissent une fonction intraspécifique chez les pucerons ou entrent dans le mécanisme de reconnaissance de l'hôte. Cependant leur large distribution dans le règne végétal, spécialement sous forme estérifiée (THOMPSON, 1980), peut justifier leur intégration parmi les allélochimiques. GREENWAY et al. (1978) ont testé une série de 50 acides carboxyliques, saturés ou non, et quelques dérivés. En situation de choix, les acides monocarboxyliques sont nettement refusés (de $\mathrm{C}_{8}$ à $\mathrm{C}_{13}$ ) ou légèrement acceptés (de $\mathrm{C}_{17}$ à $\mathrm{C}_{21}$ ) par $M$. persicae, les acides dicarboxyliques de même sont légèrement acceptés. L'insaturation n'a guère d'effet.

Des études plus précises révèlent que l'acide dodécanoïque (ou acide laurique) (10, fig. 1) et l'acide undécanoïque réduisent le nombre et la durée moyenne des piqûres de $M$. persicae, sur « feuille artificielle » (céramique + parafilm) (PHELAN \& MIL LER, 1982) et sur feuille de Brassica pekinensis Rupr. (SHERWOOD et al., 1981). Toutefois ces 2 acides n'empêchent pas le comportement d'épreuve d'ailés de $M$. persicae après atterrissage sur «feuille artificielle ", mais inhibent la fixation définitive du puceron (PHELAN \& MiLLER, 1982). L'acide heptadécanoïque encourage la fixation de $M$. persicae sur membrane de parafilm (GREENWAY et al., 1978), mais n'altère pas son comportement d'épreuve sur « feuille artificielle » (PHELAN \& MiLler, 1982). Enfin, l'acide tétracosanoïque (ou lignocérique), mais non l'acide octocosanoïque, réduit la durée de la $1^{\text {re }}$ piqûre d'épreuve d'Acyrthosiphon pisum (KLINGAUF, 1972).

L'acide dodécanoïque inhibe l'acquisition par $M$. persicae de 4 virus : 2 persistants (PLRV = potato leafroll virus, virus de l'enroulement de la pomme de terre ; BMYV = beet mild yellowing virus, virus de la jaunisse modérée de la betterave), un semi-persistant (BYV = beet yellows virus, virus de la jaunisse grave de la betterave) et un à transmission bimodale (CaMV = cauliflower mosaic virus, virus de la mosaïque du chou-fleur). L'acquisition du virus non-persistant PVY (potato virus $Y$, virus $Y$ de la pomme de terre) est favorisée au contraire par l'acide dodécanoïque (GIBSON et al., 1982). Ces effets peuvent s'expliquer par l'inhibition du comportement d'épreuve que provoque l'acide dodécanoïque. En effet, l'acquisition de virus non-persistants est d'autant plus efficient que les piqûres sont plus brèves, alors que celle des autres types de virus exige des piqûres de longue durée (LECLANT, 1968).

\section{E. Alcaloïdes}

Nombreux sont les alcaloïdes, souvent sous forme de glycosides, dont l'activité antiappétante sur différents insectes vivant sur solanacées a été recherchée (Munakata, 1977 ; Vigneron, 1978). Quant aux pucerons, SCHOONHOVEN \& DERKSEN-KOPPERS (1976) signalent qu'en situation de choix $M$. persicae refuse un aliment synthétique contenant un alcaloïde (atropine, berbérine, caféine, capsaïcine, conessine, hordénine, quinine). La solanine ( 11 , fig. 1) et plus encore la tomatine (12, fig. 1), respectivement trouvées dans la pomme de terre et la tomate, solanacées hôtes pour $M$. persicae, sont relativement acceptables, sans être phagostimulantes. La polyphagie de $M$. persicae serait liée, hormis le rôle de la qualité nutritive de l'hôte, à la tolérance de cet insecte à de nombreux allélochimiques. Il réagit toutefois à quelques phagodissuadants (SCHOONHOVEN \& DERKSEN-KOPPERS, 1976 ; QIN \& KE, 1984).

Un alcaloïde, la spartéine (13, tig. 1) extraite du genêt à balais (Sarothamnus scoparius L.), est connu pour stimuler l'alimentation d'Acyrthosiphon spartii (Koch) et jouer le rôle de stimulus spécifíque dans la sélection de l'hôte (SMITH, 1966). Alcaloïde antiappétant pour différents insectes, la zanthophylline ne semble pas atfecter la prise alimentaire de $S$. graminum (CAPINERA \& STERMITZ, 1979). Enfin, la résistance de Lupinus spp. à $A$. pisum (WEGOREK \& KRZYMAŃSKA, 1971) et Macrosiphum euphorbiae (Thomas) (BRUSSE, 1962) a été attribuée à des concentrations élevées d'alcaloïdes. 


\section{F. Substances phénoliques et flavonoïdes}

En solution nutritive, des composés phénoliques extraits de tissus superficiels de Vicia faba et de Beta vulgaris agissent comme phagostimulants sur $A$. fabae, ainsi que 13 phénols simples (mono-, di-, triphénols, phénolamines, flavonoïdes) (JÖRDENSRÖTTGER, 1979b).

Diverses substances phénoliques sont impliquées dans la résistance variétale des céréales aux pucerons ; ainsi la résistance du blé d'hiver à $R$. padi et Sitobion avenae (F.) a été associée à des teneurs élevées en monophénols (NIRAZ \& DABrowSKI, 1980), celle de l'orge à $S$. graminum a été attribuée à divers phénols et dérivés (ToDD et al., 1971), dont l'alcool benzylique (14, fig. 1) (JunEja et al., 1972, 1975). Si plusieurs de ces composés agissent par toxicité sur les pucerons (TODD et al., 1971; SCHOONHOVEN \& DERKSEN-KOPPERS, 1976), l'activité phagodissuadante de certains phénols et flavonoïdes sur $M$. persicae et S. graminum est bien établie (DREYER et al., 1981 ; DREYER \& JONES, 1981); le rôle de ces substances dans le comportement de sélection et d'alimentation de ces pucerons reste toutefois à élucider.

Des roténones extraites d'Amorpha fruticosa L. (Légumineuses), antiappétantes pour divers insectes, sont sans effet sur Megoura viciae (GOMBOS \& GASKó, 1977). Enfin signalons qu'il existe une corrélation inverse entre la probabilité d'induction d'une galle par Pemphigus betae (Doane) sur Populus angustifolia James et la teneur totale en phénol des différentes parties de l'arbre (ZUCKER, 1982).

\section{G. Terpénoïdes}

De nombreux composés terpéniques (mono-, sesqui-, di- et triterpènes), capables de modifier le comportement alimentaire des insectes phytophages, ont été isolés de plantes généralement tropicales. L'inventaire qu'en donne Vigneron (1978) rend compte de la variété de ces substances, tant dans leur nature chimique que dans leur spécificité d'action. Dans l'état actuel des recherches, nous disposons de peu de données concernant l'action des terpénoïdes sur les pucerons. Des études électrophysiologiques ont mis en évidence l'effet excitateur ou inhibiteur de quelques terpènes volatils sur les récepteurs sensoriels des antennes de Nasonovia ribisnigri (Mosley) (BROMLEY \& ANDERSON, 1982) ; la signification biologique de ces effets reste encore mal comprise.

Chapman et al. (1981) ont montré que les ailés de Cavariella aegopodii (Scopoli) sont fortement attirés par l'odeur du carvone (15, fig. 1), monoterpène extrait de diverses ombellifères hôtes pour ce puceron. Inversement, le linalool (16, fig. 1) réduit, selon ces auteurs, les effectifs des captures de ce même puceron, soit par inhibition de l'atterrissage, soit par altération du comportement d'orientation.

Le polygodial (17, fig. 1), sesquiterpène isolé de Polygonum hydropiper L. (Polygonacées), présente une forte activité antiappétante sur divers lépidoptères. Son action sur $M$. persicae a été examinée par GiBSON et al. (1982), en considérant en particulier le pouvoir virulifère de ce puceron. Le polygodial inhibe la fixation et le dépôt de larves de $M$. persicae sur des feuilles coupées de différentes plantes-hôtes ainsi que l'acquisition du BYV et du PVY. Son action sur l'acquisition de ce dernier virus, non-persistant, laisse à penser que le polygodial pourrait supprimer le comportement d'épreuve. Or, il semble bien établi que cet allélochimique n'empêche pas des ailés de $M$. persicae de piquer (GRIFFITHS et al., 1982). Les modalités de l'inhibition du PVY par le polygodial restent méconnues et sont à rechercher.

ROSE et al. (1981) ont examiné l'effet sur S. graminum d'une série de 11 acides diterpéniques extraits de la composée Grindelia humulis Hook. \& Arn. Des réponses variables ont été obtenues; ainsi l'acide hydroxy-18 grindélique ( 18 , fig. 1) s'est montré le plus actif, avec une $\mathrm{ED}_{50}$ de 0,002 p. 100 , alors que l'acide kaurén-16 ö̈que (19, fig. 1$)$ est inactif à 1 p. 100 .

Les triterpènes isolés de plantes tropicales (Méliacées, Apocynacées, Simarubacées, ...) présentent un intérêt considérable pour le biologiste et le chimiste, par leur puissant pouvoir antiappétant, leur spectre d'activité et leur variété (JACOBSON, 1981). L'une des molécules les plus actives, l'azadirachtine (20, fig. 1), est extraite de la méliacée $A$ zadirachta indica A. Juss. Si son activité antiappétante sur des insectes appartenant à divers ordres est bien connue et documentée (SCHMUTTERER et al., 1981), son action sur les pucerons reste mal établie :

- plusieurs extraits de graines d' $A$. indica se révèlent toxiques pour Rhopalosiphum nymphaeae (L.) (GoYAl et al., 1971) ;

- Gill (1972) (cité par CHAPMAN, 1974) affirme que l'azadirachtine, absorbée par la plante-hôte, est sans effet sur la prise alimentaire de $M$. persicae;

- une solution nutritive contenant de l'azadirachtine $0,001 \mathrm{M}$ est refusée par $M$. persicae en situation de choix et provoque une mortalité de 100 p. 100 en $2 \mathrm{j}$ en non-choix (SCHOONHOVEN \& DERKSEN-KOPPERS, 1976) :

- des extraits de chloroforme-méthanol d' $A$. indica, déposés sur une ou entre 2 membranes de parafilm, ou incorporés dans un aliment synthétique, réduisent la fixation et la reproduction de $M$. persicae. Déposés sur des feuilles de Brassica oleracea var. acephala D.C., ces extraits limitent la colonisation aphidienne; absorbés par la plante, ils engendrent le même effet, mais seulement à des concentrations phytotoxiques (GRIFFITHS et al., 1978).

\section{H. Divers}

Caractéristique des légumineuses, la coumarine (21, fig. 1) à haute concentration inhibe la prise alimentaire de certains coléoptères vivant normalement sur légumineuses (CHAPMAN, 1974). Toutefois son effet sur l'alimentation des pucerons est mal défini (HowE \& GORZ, 1960 ; MANSOUR et al., 1982).

La reconnaissance de $V$. faba par $A$. pisum fait intervenir un alcane linéaire de formule brute n- $\mathrm{C}_{32} \mathrm{H}_{66}$ présent dans la cuticule de la plante, et doué de propriétés phagostimulantes sur ce puceron (NÖCKER-WENZEL et al., 1971; KLINGAUF et al., 1971 ; KLINGAUF, 1972).

Des études électrophysiologiques ont montré l'effet stimulateur d'alcools et d'aldéhydes en $\mathrm{C}_{6}$ (transhexen-2 ol-1, hexénal) sur les chémorécepteurs antennaires de $S$. avenae (YAN \& VISSER, 1982) et de N. ribisnigri (BROMLEY \& ANDERSON, 1982). Ces substances entrent dans la composition des odeurs de 
la plupart des végétaux. Certains phytophages, comme le doryphore, sont attirés par l'odeur de leur plantehôte, spécialement par les composés en $\mathrm{C}_{6}$ (VISSER, 1983), et il est probable qu'une stimulation semblable existe chez les pucerons. Par ailleurs $A$. fabae est attiré par voie olfactive par des extraits de Beta vulgaris et de V. faba (ALIKHAN, 1960).

Enfin, la littérature fournit quelques cas de stimulation ou d'inhibition de la prise alimentaire des pucerons par des extraits végétaux dont le(s) principe(s) actif(s) n'a (n'ont), à notre connaissance, pas été identifié(s). Ainsi des extraits de feuilles de Fragaria vesca L. stimulent la piqûre du puceron du fraisier, Chaetosiphon fragaefolii (Cockerell) (SHANKS \& FINNIGAN, 1970). Les extraits bruts d'Equisetum arvense L. (BREMER, 1959 ; SCHMID \& HENGGELER, 1982) et de Foeniculum vulgare Mill. (WENSLER, 1962) seraient répulsifs. Par marquage radioactif, on a montré que des extraits de Tripsacum floridanum Porter et de Raphanus sativus L. réduisent l'ingestion d'une solution de saccharose par $R$. maidis (GUSS \& BRANSON, 1972 ) et par $M$. persicae (DUFFUS \& GOLD, 1967), respectivement. Des extraits bruts d'Allium sativum L. entraînent une mortalité accrue de $S$. avenae et $R$. padi sur avoine, et de $M$. persicae sur betterave (NASSEH, 1983). Des pucerons se développant normalement sur Cuscuta campestris Yuncker refusent cette plante si elle parasite l'oignon (HARVEY, 1966) ; le rôle d'allélochimiques dans ce phénomène n'est pas à exclure.

\section{CONCLUSIONS}

A la lumière des travaux entrepris et des résultats publiés, le rôle des allélochimiques dans les relations plantes-pucerons reste mal compris, hormis quelques exemples particuliers.

A. Les phases successives du comportement de sélection de l'hôte et d'alimentation des pucerons sont sous la dépendance de stimuli de divers ordres, dont l'intégration par le puceron déterminera sa réponse : rejet de la plante ou poursuite du comportement. Nous avons vu que des allélochimiques jouent un rôle à différents niveaux du comportement du puceron et peuvent être déterminants dans la spécificité des relations puceron-plante.

L'idée générale, selon laquelle le puceron ailé ne reconnaît pas sa plante-hôte avant l'atterrissage (KENNEDY et al., 1959), doit être nuancée pour certaines espèces dont le vol peut être orienté par un stimulus émanant de l'hôte. C'est le cas de C. aegopodii, attiré par l'odeur d'un allélochimique, le carvone (cf. G) ainsi que probablement de $S$. avenae attiré par des composés volatils en $\mathrm{C}_{6}$ (cf. $\mathrm{H}$ ).

Le rôle des allélochimiques dans le comportement alimentaire des pucerons n'est établi que dans le cas de quelques espèces. La littérature fournit des exemples d'allélochimiques intervenant sur :

- le comportement d'épreuve : la phlorizine (cf. $C_{1}$ ), la sinigrine (cf. $C_{2}$ ), la spartéine (cf. $E$ ) ;

- le comportement de pénétration des stylets, par stimulation : la sinigrine pour Lipaphis erysimi (cf. $\mathrm{C}_{2}$ ), ou par inhibition : la phlorizine (cf. $\mathrm{C}_{1}$ ) ;
- le comportement d'ingestion : les allélochimiques nutritifs (saccharose et aminoacides).

VAN EMDEN (1978) attire l'attention sur la difficulté d'associer un allélochimique unique et un effet comportemental donné. Un même composé peut déclencher des réponses différentes selon sa concentration ou, à même concentration, selon la teneur qualitative en autres substances. Est déterminante pour le comportement du puceron l'« image phytochimique totale " (total phytochemical picture, VAN EMDEN, 1973). Les interactions entre nutriments et allélochimiques s'avèrent complexes. Selon VAN EMDEN, les allélochimiques participent non seulement à la valeur phagostimulante de la plante-hôte (balance phagostimulants/antiappétants), mais encore à sa qualité nutritive, en agissant sur les quantités de nourriture et, de ce fait, sur les performances du puceron.

B. Du point de vue des relations plantes-pucerons, les allélochimiques passés en revue agissent soit par stimulation, soit par inhibition, une même substance pouvant présenter les 2 effets sur des espèces différentes. La réponse des pucerons aux allélochimiques dépend de leur spécificité d'hôte. Bien que pour les pucerons il s'avère prématuré de dégager des tendances générales, il apparaît cependant que les espèces mono- et oligophages sont plus sensibles aux inhibiteurs que les espèces polyphages. Cette constatation confirme les tendances formulées par JERMY (1966) pour les insectes broyeurs. D'autre part, les cas connus d'allélochimiques agissant comme stimuli spécifiques de reconnaissance de l'hôte (sinigrine, phlorizine, spartéine) concernent des espèces mono- et oligophages inféodées aux plantes contenant ces substances. Généralement ces mêmes substances sont inhibitrices pour les espèces non inféodées à ces plantes, la réponse étant la plus forte chez les espèces mono- et oligophages.

C. Les recherches sur les interactions chimiques entre plantes et pucerons permettent d'envisager une prise en compte de ces mécanismes dans les stratégies de lutte antiaphidienne. En vue de réduire la colonisation des plantes cultivées et la transmission de virus phytopathogènes, la solution idéale serait d'orienter le comportement de sélection du puceron vers le refus de la plante-hôte, voire d'empêcher l'atterrissage. des ailés, au moyen de répulsifs. Mais déjà serait-il intéressant, à l'aide de ces substances et en combinaison avec d'autres méthodes de lutte, de pouvoir freiner la colonisation des plantes cultivées et maintenir les populations aphidiennes en deçà du seuil de tolérance. Il en résulterait une limitation des dégâts directs et indirects, ainsi qu'un possible déplacement des équilibres vers une augmentation de l'efficacité des auxiliaires.

Au sujet de l'effet des allélochimiques sur la transmission de virus, les résultats obtenus avec l'acide dodécanoïque (cf. D) et le polygodial (cf. G) montrent que l'action d'antiappétants sur les pucerons peut entraîner une réduction de l'acquisition de virus persistants et semi-persistants. Quant aux virus nonpersistants, leur taux de transmission peut être favorisé par des allélochimiques, comme l'acide dodécanoïque, qui réduisent la durée des piqûres d'épreuve. Cependant, on l'a vu, le polygodial se révèle capable 
de limiter la transmission du PVY, non-persistant, selon des modalités encore inexpliquées. Ainsi le concept de répulsifs capables de limiter la transmission, même de virus non-persistants, peut être soutenu. L'emploi de répulsifs présente toutefois l'inconvénient de stimuler la mobilité des pucerons, ce qui peut favoriser la propagation de viroses. De nombreux travaux, tant écologiques qu'éthologiques et physiologiques, sont encore nécessaires, afin d'approfondir nos connaissances sur les relations allélochimiques - transmission de virus, avant toute possibilité d'intégration de ces substances dans les programmes de lutte.

Reçu le 21 juin 1984.

Accepté le 5 décembre 1984.

\section{REMERCIEMENTS}

Ce travail a été réalisé à l'U.S.T.L.-Montpellier dans le cadre d'un doctorat de $3^{\text {e }}$ cycle soutenu par une allocation D.G.R.S.T J'exprime ma gratitude aux Docteurs P. C. ROBERT, Y. BOUCHERY, B. Muckensturm, et à M. le Professeur F. Leclant, pour la correction du manuscrit, leurs encouragements et les suggestions apportées.

\section{RÉFÉRENCES BIBLIOGRAPHIQUES}

Alikhan M. A., 1960. The experimental study of the chemotactic basis of host-specificity in a phytophagous insect, Aphis fabae Scop. (Aphididae: Homoptera). Ann. Univ. Mariae CurieSklodowska, Sect. C: Biol., 15, 117-158.

Argandoña V. H., Luza J. G., Niemeyer H. M., Corcuera L. J., 1980. Role of hydroxamic acids in the resistance of cereals to aphids. Phytochemistry, 19, 1665-1668.

Argandoña V. H., Niemeyer H. M., Corcuera L. J., 1981. Effect of content and distribution of hydroxamic acids in wheat on infestation by the aphid Schizaphis graminum. Phytochemistry, 20, 673-676.

Argandoña V. H., Corcuera L. J., Niemeyer H. M., Campbell B. C., 1983. Toxicity and feeding deterrency of hydroxamic acids from Gramineae in synthetic diets against the greenbug, Schizaphis graminum. Entomol. Exp. Appl., 34, 134-138.

Arn H., Cleere J. S., 1971. A double-label choice-test for the simultaneous determination of diet preference and ingestion by the aphid Amphorophora agathonica. Entomol. Exp. Appl., 14, 377 387.

Auclair J. L., 1969. Nutrition of plant-sucking insects on chemically defined diets. Entomol. Exp. Appl., 12, 623-641.

Bernays E. A., Simpson S. J., 1982. Control of food intake. $A d v$. Insect Physiol., 16, 59-118.

Bremer H., 1959. Versuche zur Abschreckung von Blattläusen. Anz. Schaedlingskd., 32, 17-18.

Bromley A. K., Anderson M., 1982. An electrophysiological study of olfaction in the aphid Nasonovia ribis-nigri. Entomol. Exp. Appl., 32, 101-110.

Brusse M. J., 1962. Alkaloid content and aphid infestation in Lupinus angustifolius L. N. Z. J. Agric. Res., 5, 188-189.

Capinera J. L., Stermitz F. R., 1979. Laboratory evaluation of zanthophylline as a feeding deterrent for range caterpillar, migratory grasshopper, alfalfa weevil, and greenbug. J. Chem. Ecol., 5, 767-771.

Chapman R. F., 1974. The chemical inhibition of feeding by phytophagous insects : a review. Bull. Entomol. Res., 64, 339-363.

Chapman R. F., Bernays E. A., Simpson S. J., 1981. Attraction and repulsion of the aphid, Cavariella aegopodii, by plant odors. $J$. Chem. Ecol., 7, 881-888.

Corcuera L. J., Argandoña V. H., Peña G. F., Perez F. J., Niemeyer H. M., 1982. Effect of benzoxazinone from wheat on aphids. Proc. 5th int. Symp. Insect-Plant Relationships, Wageningen, 1-4 mars 1982, 33-39.

Dadd R. H., Krieger D. L., 1968. Dietary amino acid requirements of the aphid, Myzus persicae. J. Insect Physiol., 14, 741-764.

Dreyer D. L., Jones K. C., 1981. Feeding deterrency of flavonoids and related phenolics towards Schizaphis graminum and Myzus persicae : aphid feeding deterrents in wheat. Phytochemistry, 20, 2489-2493.
Dreyer D. L., Reese J. C., Jones K. C., 1981. Aphid feeding deterrents in sorghum. Bioassay, isolation, and characterization. $J$. Chem. Ecol., 7, 273-284.

Duffus J. E., Gold A. H., 1967. Relationship of tracer-measured aphid feeding to acquisition of beet western yellows virus and to feeding inhibitors in plant extracts. Phytopathology, 57, 1237-1241.

Gibson R. W., Rice A. D., Pickett J. A., Smith M. C., Sawicki R. W., 1982. The effects of the repellents dodecanoic acid and polygodial on the acquisition of non-, semi- and persistent plant viruses by the aphid Myzus persicae. Ann. Appl. Biol., 100, 55-59.

Gombos M. A., Gaskó K., 1977. Extraction of natural antifeedants from the fruits of Amorpha fruticosa L. Acta Phytopathol. Acad. Scient. Hung., 12, 349-357.

Goyal R. S., Gulati K. C., Sarup P., Asim Kidwai M., Singh D. S., 1971. Biological activity of various alcohol extractives and isolates of neem (Azadirachta indica) seed cake against Rhopalosiphum nympheae (Linn.) and Schistocerca gregaria Horsk. Indian I. Entomol., 33, 67-71.

Greenway A. R., Griffiths D. C., Lloyd S. L., 1978. Response of Myzus persicae to components of aphid extracts and to carboxylic acids. Entomol. Exp. Appl., 24, 369-374.

Griffiths D. C., Greenway A. R., Lloyd S. L., 1978. The influence of repellent materials and aphid extracts on settling behaviour and larviposition of Myzus persicae (Sulzer) (Hemiptera, Aphididae). Bull. Entomol. Res., 68, 613-619.

Griffiths D. C., Pickett J. A., Woodcock C., 1982. Behaviour of alatae of Myzus persicae (Sulzer) (Hemiptera, Aphididae) on chemically treated surfaces after tethered flight. Bull. Entomol. Res., 72, 687-693.

Guss P. L., Branson T. F., 1972. The use of 75 Se in feeding studies with the corn leaf aphid (Hemiptera (Homoptera), Aphididae). Ann. Entomol. Soc. Am., 65, 303-306.

Harrewijn P., Noordink J. P. W., 1971. Taste perception of Myzus persicae in relation to food uptake and developmental processes. Entomol. Exp. Appl., 14, 413-419.

Harvey T. L., 1966. Aphids, dodder (Cuscuta campestris), and dodder-host plant interrelations. Ann. Entomol. Soc. Am., 59, 1276-1282 (In : Rev. Appl. Entomol., 1967, 55, 360).

Herrbach E., 1985. Rôle des sémiochimiques dans les relations pucerons-plantes I. Généralités et phéromones d'alarme. Agronomie, 5 (3).

Howe W. L., Gorz H. J., 1960. Feeding preferences of the cowpea aphid among species of Melilotus. Ann. Entomol. Soc. Am., 53, 696-697 (In : Rev. Appl. Entomol., 1961, 49, 549).

Jacobson M., 1981. Isolation and identification of insect antifeedants and growth inhibitors from plants : an overview, p. 13-19. In H. Schmutterer, K. R. S. Ascher, H. Rembold, ed., 1981, q.v.

Jermy T., 1966. Feeding inhibitors and food preference in chewing phytophagous insects. Entomol. Exp. Appl., 9, 1-12. 
Jördens D., Klingauf F., 1977. Der Einfluss von L-Dopa auf Ansiedlung und Entwicklung von Aphis fabae Scop. an synthetischer Diät. Meded. Fac. Landbouwwet. Rijksuniv. Gent, 42, 14111419 .

Jördens-Röttger D., 1979a. Das Verhalten des Schwarzen Bohnenblattlaus, Aphis fabae Scop. gegenüber chemischen Reizen von Pflanzenoberflächen. Z. angew. Entomol., 88, 158-166.

Jördens-Röttger D., 1979b. The role of phenolic substances for host-selection behaviour of the black bean aphid, Aphis fabae. Entomol. Exp. Appl., 26, 49-54.

Juneja P. S., Gholson R. K., Burton R. L., Starks K. J., 1972. The chemical basis for greenbug resistance in small grains. I. Benzyl alcohol as a possible resistance factor. Ann. Entomol. Soc. Am., 65, 961-964.

Juneja P. S., Pearcy S. C., Gholson R. K., Burton R. L., Starks K. J., 1975. Chemical basis for greenbug resistance in small grains. II. Identification of the major neutral metabolite of benzyl alcohol in barley. Plant Physiol., 56, 385-389.

Kennedy J. S., Booth C. O., Kershaw W. J. S., 1959. Host finding by aphids in the field. I. Gynoparae of Myzus persicae (Sulzer). Ann. Appl. Biol., 47, 410-423.

Klingauf F., 1971. Die Wirkung des Glucosids Phlorizin auf das Wirtswahlverhalten von Rhopalosiphum insertum (Walk.) und Aphis pomi De Geer (Homoptera, Aphididae). Z. Angew. Entomol., 68, 41-55.

Klingauf F., 1972. Die Bedeutung von peripher vorliegenden Pflanzensubstanzen für die Wirtswahl von phloemsaugenden Blattläusen (Aphididae). Z. Pflanzenkr. Pflanzenschutz, 79, 471-477.

Klingauf F., Nöcker-Wenzel K., 1972. Einfluss von Aminosäuren auf das Wirtswahlverhalten von Acyrthosiphon pisum (Homoptera, Aphididae) unter besonderer Berücksichtigung ihres chemischen Aufbaus. Entomol. Exp. Appl., 15, 274-286.

Klingauf F., Nöcker-Wenzel K., Klein W., 1971. Einfluss einiger Wachskomponenten von Vicia faba $\mathrm{L}$. auf das Wirtswahlverhalten von Acyrthosiphon pisum (Harris) (Homoptera, Aphididae). Z. Pflanenkr. Pflanzenschutz, 78, 641-648.

Klingauf F., Sengonca C.., Bennewitz H., 1972. Einfluss von Sinigrin auf die Nahrungsaufnahme polyphager und oligophager Blattlausarten (Aphididae). Oecologia, 9, 53-57.

Kunkel H., 1977. Membrane feeding systems in aphid research, 311338. In K. F. Harris, K. Maramorosch: "Aphids as virus vectors ". Academic Press, New York, $559 \mathrm{p}$.

Leckstein P. M., Llewellyn M., 1974. The role of amino acids in diet uptake and selection and the utilization of dipeptides by Aphis fabae. J. Insect Physiol., 20, 877-885.

Leclant F., 1968. Connaissances actuelles sur les pucerons dans leurs relations avec les maladies à virus des plantes. Ann. Epiphyt., 19, 455-482.

Lérin J., 1980. Influence des substances allélochimiques des Crucifères sur les insectes. Acta oecol. Oecol. Gen., 1, 215-235.

Long B. J., Dunn G. M., Bowman J. S.. Routley D. G., 1977. Relationship of hydroxamic acid content in corn and resistance to the corn leaf aphid. Crop Sci., 17, 55-58.

Mac Lean D. L., Kinsey M. G., 1964. A technique for electronically recording aphid feeding and salivation. Nature, 202, 1358-1359.

Mansour M. H., Dimetry N. Z., Rofaeel I. S., 1982. The role of coumarin as secondary plant substance in the food specificity of the cowpea aphid Aphis craccivora Koch. Z. Angew. Entomol., 93, 151-157.

Masson C., 1982. Apports de la neurophysiologie pour l'identification des médiateurs chimiques par les insectes. Symp. int. Médiateurs chimiques, Versailles, 16-20 nov. 1981. Les Colloques de I'INRA, 7, 19-30.

Massonié G., 1973. Elevage d'un biotype de Myzus persicae Sulzer sur milieu synthétique. II. - Influence de la qualité de l'alimentation azotée sur la biologie du puceron. Ann. Lool. Ecol. anim., 5, 559568.

Mittler T. E., 1967a. Effect of amino acid and sugar concentration on the food intake of the aphid Myzus persicae. Entomol. Exp. Appl., 10, 39-51.

Mittler T. E., 1967b. Gustation of dietary amino acids by the aphid Myzus persicae. Entomol. Exp. Appl., 10, 87-96.
Mittler T. E., 1967c. Effect on aphid feeding of dietary methionine. Nature, 214, 386.

Mittler T. E., 1970. Effects of dietary amino acids on the feeding rate of the aphid Myzus persicae. Entomol. Exp. Appl., 13, 432-437.

Mittler T. E., Dadd R. H., 1964. Gustatory discrimination between liquids by the aphid Myzus persicae (Sulzer). Entomol. Exp. Appl., 7, 315-328.

Mittler T. E., Dadd R. H., 1965. Differences in the probing responses of Myzus persicae (Sulzer) elicited by different feeding solutions behind a parafilm membrane. Entomol. Exp. Appl., 8, 107-122.

Mittler T. E., Dadd R. H., Daniels S. C. Jr., 1970. Utilization of different sugars by the aphid Myzus persicae. J. Insect Physiol., 16, 1873-1890.

Montgomery M. E., 1975. Feeding of the aphids Amphorophora agathonica Hottes, Aphis pomi De Geer, and Myzus persicae (Sulzer) on synthetic diets in relation to host plant selection. Diss. Abstr. int. B. Sci. Eng., 35, 3370-B.

Montgomery M. E., Arn H., 1974. Feeding response of Aphis pomi, Myzus persicae and Amphorophora agathonica to phlorizin. J. Insect Physiol., 20, 413-421.

Moon M. S., 1967. Phagostimulation of a monophagous aphid. Oikos, 18, 96-101.

Munakata K., 1977. Insect feeding deterrents in plants, 93-102. In H. H. Shorey, J. J. McKelvey : "Chemical control of insect behavior ". J. Wiley \& Sons, New York, 414 p.

Nasseh M. O., 1983. Wirkung von Rohextrakten aus Allium sativum L. auf Getreideblattläuse Sitobion avenae F. und Rhopalosiphum padi L. sowie die Grüne Pfirsichblattlaus Myzus persicae Sulz. Z. angew. Entomol., 95, 228-230.

Nault L. R., Styer W. E., 1972. Effects of sinigrin on host selection by aphids. Entomol. Exp. Appl., 15, 423-437.

Niraz S., Dabrowski Z., 1980. The mechanisms involved in the sensivity of winter wheat to aphids infestation. Eucarpia Congress "Genetic Resource and Plant Breeding for Resistance ", Leningrad, 3, 42-43.

Nöcker-Wenzel K., Klein W., Klingauf F., 1971. Isolierung von Oberflächensubstanzen aus Vicia faba L. im Rahmen von Untersuchungen zur Insekt-Wirtsptlanzen-Beziehung. Tetrahedron Lett., $\mathrm{n}^{\circ} 46,4409-4412$.

Pettersson J., 1973. Olfactory reactions of Brevicoryne brassicae (L.) (Hom., Aph.). Swed. J. Agric. Res., 3, 95-103.

Pettersson J., 1979. Aphids and host plant communication. Symb. Bot. Ups., 22, 107-113.

Phelan P. L., Miller J. R., 1982. Postlanding behaviour of alate Myzus persicae as altered by (E)- $\beta$-farnesene and three carboxylic acids. Entomol. Exp. Appl., 32, 46-53.

Qin J., Ke L., 1984. The influence of secondary plant substances on the growth and development of Myzus persicae of Beijing. Entomol. Exp. Appl., 35, 17-20.

Rose A. F., Jones K. C., Haddon W. F., Dreyer D. C., 1981. Grindelane diterpenoid acids from Grindelia humulis: feeding deterrency of diterpene acids towards aphids. Phytochemistry, 20, 2249-2255.

Schmid O., Henggeler S., 1982. Ravageurs et maladies au jardin. Les solutions biologiques. Ed. Terre vivante, Paris, $225 \mathrm{p}$.

Schmutterer H., Ascher K. R. S., Rembold H., ed., 1981. Natural pesticides from the neem tree (Azadirachta indica A. Juss). Proc. 1st. Neem Conf., Rottlach-Egern, 16-18 juin 1980, 297 p.

Schoonhoven L. M., Derksen-Koppers I., 1976. Effects of some allelochemics on food uptake and survival of a polyphagous aphid, Myzus persicae. Entomol. Exp. Appl., 19, 52-56.

Shanks C. H. Jr., Finnigan B., 1970. Probing behaviour of the strawberry aphid. Ann. Entomol. Soc. Am., 63, 734-737.

Sherwood M. H., Greenway A. R., Griffiths D. C., 1981. Response of Myzus persicae (Sulzer) (Hemiptera : Aphididae) to plants treated with fatty acids. Bull. Entomol. Res., 71, 133-136.

Smith B. D., 1966. Effect of plant alkaloid sparteine on the distribution of the aphid Acyrthosiphon spartii (Koch). Nalure, 212, 213-214. 
Srivastava P. N., Auclair J. L., 1971. Influence of sucrose concentration on diet uptake and performance by the pea aphid, Acyrthosiphon pisum. Ann. Entomol. Soc. Am., 64, 739-743.

Srivastava P. N.. Auclair J. I... 1974. Eflect of amino acid concentration on diet uptake and performance by the pea aphid, Acyrthosiphon pisum (Homoptera, Aphididae). Can. Entomol., 106, 149-156.

Thompson G. A. Jr., 1980. Plant lipids of taxonomic significance. Encycl. Plant Physiol. (N.S.), 8, 535-553.

Todd G. W., Getahun A., Cress D. C., 1971. Resistance in barley to the greenbug, Schizaphis graminum. I. Toxicity of phenolic and flavonoid compounds and related substances. Ann. Entomol. Soc. Am., 64, 718-722.

Underhill E. W., 1980. Glucosinolates. Encycl. Plant Physiol. (N.S.), 8, 493-511.

Van Emden H. F., 1972. Aphids as phytochemists, 25-43. In J. B. Harborne : «Phytochemical Ecology». Academic Press, New York, 272 p.

Van Emden H. F., 1973. Aphid host plant relationships. Some recent studies. In A. D. Lowe : «Perspectives in aphid biology». Bull. Entomol. Soc. N. Z., 2, 54-64.

Van Emden H. F., 1978. Insects and secondary plant substances. An alternative viewpoint with special reference to aphids, 309-323. In J. B. Harborne : «Biochemical aspects of plant and animal coevolution ». Academic Press, New York, 435 p.
Van Emden H. F., Bashford M., 1971. The performance of Brevicoryne brassicae and Myzus persicae in relation to leaf age and leaf amino acids. Entomol. Exp. Appl., 14, 349-360.

Vigneron J. P., 1978. Substances antiappétantes d'origine naturelle. Ann. Zool. Ecol. Anim., 10, 663-694.

Visser J. H., 1983. Differential sensory perceptions of plant compounds by insect. Meet. Ann. Chem. Soc. Plant Resistance to Insects, Las Vegas, 1982. ACS Series, n 208, 215-230.

Wearing C. H., 1968. Responses of aphids to pressure applied to liquid diet behind parafilm membrane. Longevity and larviposition of Myzus persicae (Sulz.) and Brevicoryne brassicae (L.) (Homoptera, Aphididae) feeding on sucrose and sinigrin solutions. N. Z.J. Sci., 11, 105-121.

Węgorek W., Krzymańska J., 1971. Dalsze badania nad odpornościa kubinu na mszycę grochowa (Acyrthosiphon pisum Harris). Pr. Nauk. Inst. Ochr. Rośl., 13, 7-23.

Wensler R. J. D., 1962. Mode of host selection by an aphid. Nature, 195, 830-831.

Whittaker R. H., Feeny P., 1971. Allelochemics : chemical interactions between species. Science, 171, 757-770.

Yan F. S., Visser J. H., 1982. Electroantennogram responses of the cereal aphid Sitobion avenae to plant volatile components. Proc. 5th int. Symp. Insect-Plant Relationships, Wageningen, 1-4 mars 1982, 387-388.

Zucker W. V., 1982. How aphids choose leaves: the role of phenolics in host selection by a galling aphid. Ecology, 63, 972-981. 